\title{
Impact of Buffer Map Cheating on the Streaming Quality in DONet*
}

\author{
Yong Cui, Dan Li, and Jianping $\mathrm{Wu}$ \\ Department of Computer Science, Tsinghua University, Beijing, 100084, China \\ cy@csnet1.cs.tsinghua.edu.cn, lidan@csnet1.cs.tsinghua.edu.cn, \\ jianping@cernet.edu.cn
}

\begin{abstract}
Data-driven Overlay Network (DONet) is especially suitable for live stream in P2P environment. However, it demands the cooperation of individual nodes to exchange their buffer maps. Since these nodes are selfish and have their own interests, they might cheat about their buffer maps to reduce the forwarding burden. We analyze the impact of this kind of cheating behavior on the streaming quality of DONet by experiment in this paper. The experimental results show that buffer map cheating has considerable negative impact on the streaming quality in DONet.
\end{abstract}

Keywords: DONet, Buffer Map Cheating, Selfish.

\section{Introduction}

Multimedia applications, especially live stream, have been used more and more in Internet. In live stream, a large number of users are interested in the real- time data from a common source. Compared to other applications, live stream demands higher network bandwidth as well as node forwarding capacity. Given the multi-receiver nature of live stream, multicast is the ideal supporting technology. Currently, there are two kinds of multicast technologies. One is realized in the network layer, named as IP multicast [1]; and the other is realized in the application layer, named as overlay multicast [2 12].

IP multicast builds the data structure on routers, which is a tree, and thus achieves high scalability and high efficiency. However, IP multicast changes the "unicast" principle of traditional Internet, and a lot of problems in it, such as member management, congestion control, and pricing model, have not been solved well yet. All these lead to the difficulty of deploying IP multicast in Internet scale.

Overlay multicast is subsequently proposed, which constructs the data structure in the application layer. Compared with IP multicast, overlay multicast is lower in efficiency, but much more deployable and flexible. Currently, there are two kinds of overlay multicast, namely, tree-based overlay multicast [2 7] and mesh-based overlay multicast [8 12]. Like in IP multicast, the data structure in tree-based overlay multicast is also a tree, and data is propagated along the tree after its establishment. However, overlay nodes are unstable. There is a high frequency of node join, node leave, and

\footnotetext{
Supported by the National Natural Science Foundation of China (No. 60473082, No. 90104002).
} 
node crash in overlay network. Thus, the application-layer multicast tree might change from time to time, which will bring negative impact on live stream applications that have stringent demands on the streaming continuity.

Mesh-based overlay multicast is considered as a better choice to support live stream. In mesh-based overlay multicast, the data structure is no longer a tree, but a mesh. Data-driven Overlay Network (DONet) is a representative of this kind of protocols [8]. In DONet, the stream propagated in the overlay network is divided into multiple segments. Each node maintains a number of segments, and exchanges the buffer map of available segments with partner nodes. After learning the buffer maps of partner nodes, each node requests a certain segment from a suitable partner node that holds the segment. If a node receives segment requests from partner nodes, it replies to the requests by forwarding the corresponding segments within its outgoing bandwidth. Therefore, the leave or crash of a single node will not bring too much impact on other nodes.

However, overlay nodes are not only unstable, but also selfish and strategic. Selfish nodes in DONet might cheat about their buffer maps to reduce the forwarding burden to other nodes. In such non-cooperative scenarios, some nodes cannot have the actual information to request segments, and thus the streaming quality in DONet might be impact. We try to establish the model of buffer map cheating in DONet and analyze its impact on the streaming quality by experiment in this paper, which is not studied by previous work.

\section{Related Work}

Because of the difficulty of deploying IP multicast in Internet scale, researchers turn to overlay multicast to support live stream applications. The overlay multicast protocols proposed currently can be roughly classified into two categories, namely, tree-based overlay multicast and mesh- based overlay multicast.

In tree-based overlay multicast, each node selects a long- time parent from other participating nodes to receive stream data. The parent/children relationships among all nodes compose the data structure, i.e., the multicast tree. Once the multicast tree is established, data is propagated along the tree and there is no additional control overhead. Protocols belonging to this category of overlay multicast include NARADA [2], NICE [3], ZIGZAG [4], Scattercast [5], Yoid [6], Host Multicast [7] and etc.

In mesh-based overlay multicast, there is no explicit parent/ children relationship. The data structure to propagate the stream is a mesh. Therefore, it can tolerant node dynamics well and is especially suitable to support live stream applications. Representatives of this kind of protocols include gossip-based protocols and DONet. In gossip-based protocols [9 12], each node forwards available data to a set of randomly selected nodes. But in DONet [8], each node maintains several partner nodes, and data is transmitted among partner nodes, eventually to the whole overlay network. Compared to gossip-based protocols, the advantage of DONet is that data is flowing in a request-reply way, thus there is no redundant data consuming the precious network bandwidth.

An important characteristic of overlay network is that the overlay nodes are all selfish and strategic. The selfish overlay nodes might cheat about their private information to obtain higher interests, which could affect the performance of the overall 
system. Selfish nodes in DONet might cheat about their buffer map to reduce the forwarding burden. The impact of this kind of cheating on the streaming quality in DONet is not systematically studied before, and it is discussed in this paper.

\section{Model of Buffer Map Cheating in DONet}

The stream propagated in DONet is divided into multiple segments with uniform length. A buffer map can represent the information of available segments on a node. Each node periodically exchanges its buffer map with partner nodes, and decides from which partner node to fetch a certain segment. If there are multiple partner nodes holding the same expected segment, various ways can be chosen to select the segment-providing node, for instance, the one with the shortest distance or the one with the highest outgoing bandwidth. The requests arrive at the requesting queue of the segment- providing node. When replying the requests, the segment-providing node selects some requests in the requesting queue and sends the corresponding segments within its outgoing bandwidth.

At first, we make some definitions and list the notations throughout this paper as Table 1.

Table 1. List of notations

\begin{tabular}{cl}
\hline$B$ & Buffer window size \\
$W$ & Playing waiting segment number \\
$N$ & Number of nodes in the overlay network \\
$M$ & Number of partner nodes each node maintains \\
$Q$ & Number of segments of the stream \\
$P$ & Cheating node percentage \\
$H$ & Cheating degree \\
$P c(i)$ & Playing continuity on node $i$ \\
$P d(i)$ & Playing delay on node $i$ \\
\hline
\end{tabular}

Each node in DONet maintains a buffer window, which is used to store a number of continuous segments of the stream. The buffer window size of each node is denoted as $B$. To make the streaming more continuous, each node does not begin to play the stream immediately after receiving the first segment. Instead, it waits for the arrival of the first several segments and then begins to play the stream. The number of segments each node waits for before playing the stream is defined as the playing waiting segment number, denoted by $W$. Obviously, there is $W \leq B$.

The streaming quality of each node can be measured by playing continuity and playing delay. The playing continuity of node $i$ is defined as the number of segments arriving at node $i$ no later than its playback time over the total number of segments of the stream, denoted as $P c(i)$. And the playing delay of node $i$ is defined as the average source-to-end delay of all segments playing on node $i$. Higher playing continuity and less playing delay indicate better streaming quality. The playing continuity of the DONet is defined as the average playing continuities of all nodes, and the playing delay of the DONet is defined as the average playing delays of all nodes. 
We can establish the model of buffer map cheating in DONet as follows. Suppose the number of nodes in the overlay network is $N$, the number of partner nodes each node maintains is $M$, the number of segments of the stream propagated in the overlay network is $Q$, the buffer window size is $B$, the playing waiting segment number is $W$, and the percentage of the number of cheating nodes over the total number of overlay nodes is $P$. When exchanging the buffer map with partner nodes, each cheating node hides some of its available segments, and the proportion of the number of hidden segments over the total number of available segments is $H$. This model of buffer map cheating in DONet is denoted as $C(N, M, Q, B, W, P, H)$, where $N>0, M>0,0<W \leq B \leq Q, 0 \leq P \leq 1$, and $0 \leq H \leq 1$.

\section{Experiment Study}

We conduct experiments to study the impact of buffer map cheating on the streaming quality of DONet, using the cheating model $C(N, M, Q, B, W, P, H)$ established in the last section.

Suppose the number of overlay nodes in DONet is $500(N=500)$, and the stream propagated in the network is composed of 5000 segments $(Q=5000)$. If no node cheats about their available segments, the cheating node percentage is $0 \%$. We compare the average playing continuity $(P c)$ and the average playing delay $(P d)$ of all nodes when

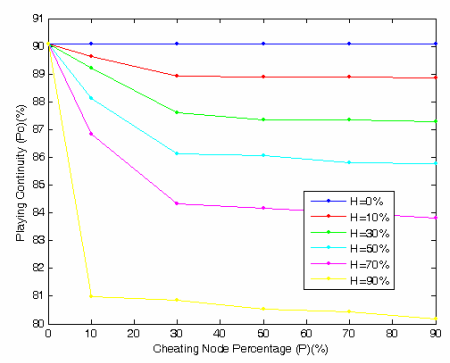

(a)

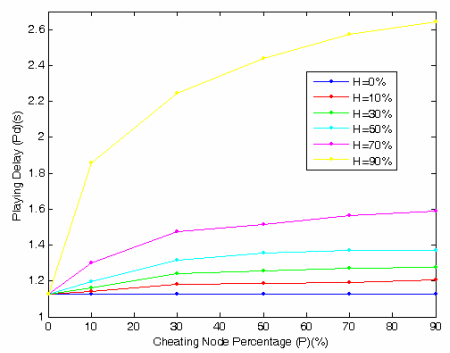

(b)

Fig. 1. Streaming quality of DONet when $M=4, B=70, W=30$. (a) playing continuity; (b) playing delay. 
the cheating node percentage $(P)$ is $0 \%$ with that of $10 \%, 30 \%, 50 \%, 70 \%$, and $90 \%$, each under different cheating degrees $(H)$, different playing waiting segment numbers $(W)$, different buffer window sizes $(B)$, and different partner numbers $(M)$.

\section{1) Different Cheating Degree}

Let $M=4, B=70, W=30$, and the cheating degree $H$ vary as $0 \%, 10 \%, 30 \%, 50 \%, 70 \%$, and $90 \%$. The playing continuity and the playing delay in these cases with different cheating node percentages are shown in Fig. 1(a) and Fig. 1(b), respectively.

From Fig. 1(a), we see that under all cheating degrees higher than $0 \%$, the maximal playing continuity is achieved when there is no node cheating. When the cheating node percentage increases, the playing continuity decreases, but the decreasing gets smoother. We also see that the playing continuity is higher with lower cheating degree. When the cheating degree is $0 \%$, which means that there is no node cheating, the playing continuity gets the optimal value. The change of playing continuity is especially steep when the cheating node percentage is low and the cheating degree is high.

Fig. 1(b) shows that under all cheating degrees more than $0 \%$, the playing delay is least when there is no node cheating. As the node cheating percentage grows, the playing delay becomes more. In addition, the lower is the cheating degree, the less is the playing delay. It is similar to Fig. 1(a) that the change of playing delay is especially obvious when the cheating node percentage is low and the cheating degree is high.

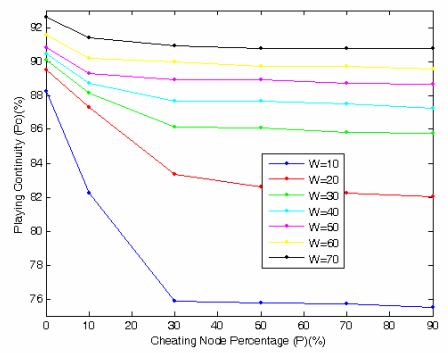

(a)

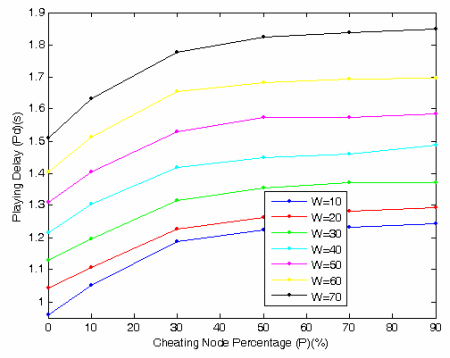

(b)

Fig. 2. Streaming quality of DONet when $M=4, B=70, H=50 \%$. (a) playing continuity; (b) playing delay. 


\section{2) Different Playing Waiting Segment Number}

Let $M=4, B=70, H=50 \%$, and the playing waiting segment number vary as $10,20,30$, $40,50,60$, and 70 . The playing continuity in these cases under different cheating node percentages is shown in Fig. 2(a), and the playing delay is shown in Fig. 2(b).

Fig. 2(a) illustrates that no matter what the playing waiting segment number is, the playing continuity is maximal when there is no node cheating. The playing continuity decreases with the growth of cheating node percentage, but the curve becomes smoother. Additionally, more playing waiting segment number brings higher playing continuity.

From Fig. 2(b), we conclude that the playing delay increases when the cheating node percentage grows, and is shortest when there is no node cheating, no matter the playing waiting segment number. Also, when the playing waiting segment number is more, the playing delay is longer.

\section{3) Different Buffer Window Size}

Let $M=4, W=30, H=50 \%$, and the buffer window size vary as $30,40,50,60,70,80$, and 90. With different cheating node percentages, the playing continuity and the playing delay are shown in Fig. 3(a) and Fig. 3(b), respectively.

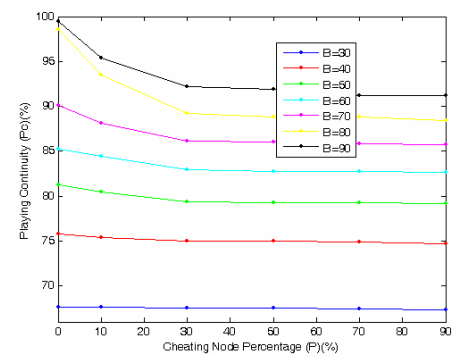

(a)

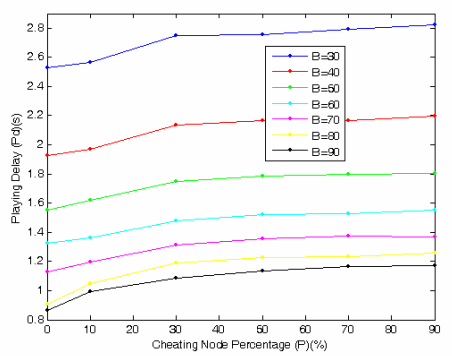

(b)

Fig. 3. Streaming quality of DONet when $M=4, W=30, H=50 \%$. (a) playing continuity; (b) playing delay.

Fig. 3(a) suggests that with all buffer window sizes, the playing continuity is highest when there is no node cheating, and gets lower with more cheating nodes. In addition, given the same cheating node percentage, the bigger buffer window size brings higher 
playing continuity. The change of playing continuity is most obvious when the buffer window size is big and the cheating node percentage is small.

Fig. 3(b) tells that the playing delay increases with the growth of cheating node percentage, and decreases with the growth of buffer window size.

\section{4) Different Partner Number}

Let $B=70, W=30, H=50 \%$, and the partner number of each node vary as 4,5 , and 6 . The playing continuity and the playing delay under different cheating node percentages are shown in Fig. 4(a) and Fig. 4(b), respectively.

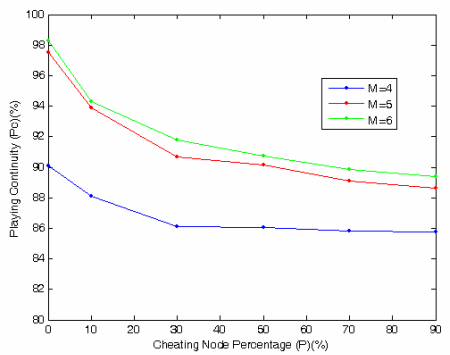

(a)

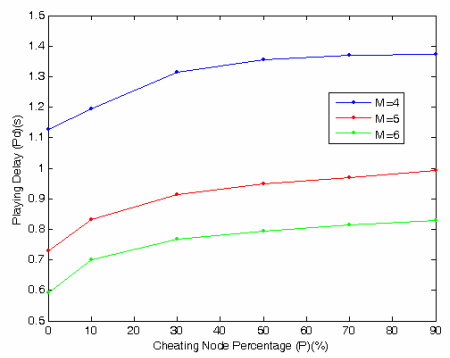

(b)

Fig. 4. Streaming quality of DONet when $B=70, W=30, H=50 \%$. (a) playing continuity; (b) playing delay.

With any number of partner nodes, the playing continuity decreases with the increase of cheating node percentage, and is maximized when there is no node cheating, as shown in Fig. 4(a). This is consistent with Fig. 1(a), Fig. 2(a), and Fig. 3(a). In addition, Fig. 4(a) shows that the playing continuity is higher when the number of partner nodes each node maintains is bigger.

On the other hand, Fig. 4(b) tells the similar conclusion as Fig. 1(b), Fig. 2(b), and Fig. 3(b), that the playing delay increases with the growth of cheating node percentage, and is shortest when there is no node cheating. Also, more partner nodes will bring shorter playing delay.

According to all the experimental results above, the streaming quality is always optimized when there is no node cheating, no matter what the network parameter is. Therefore, the buffer map cheating of selfish nodes indeed has considerable negative impact on the streaming quality of DONet. 


\section{Conclusion and Future Work}

DONet is especially suitable for live stream applications. However, selfish overlay nodes might cheat about their buffer maps to reduce the forwarding burden. We establish the model of buffer map cheating in this paper, and analyze its impact on the streaming quality of DONet by experiment in this paper. The experimental results show that buffer map cheating of selfish nodes will indeed cause the streaming quality in DONet to decrease considerably.

As for future work, we should design incentive algorithms to defend buffer map cheating in DONet, and it can be reviewed as another way to improve the streaming quality in DONet considering the selfishness of individual nodes.

\section{References}

1. S. E. Deering: Multicast Routing in Internetworks and Extended LANs, In Proceedings of ACM SIGCOMM'88, Stanford, CA, USA, (Aug 1988)

2. Y. H. Chu, S. G. Rao, and H. Zhang: A Case for End System Multicast, In Proceedings of ACM Sigmetrics'00, Santa Clara, California, USA, (Jun 2000)

3. S. Banerjee, B. Bhattacharjee, C. Kommareddy: Scalable Application Layer Multicast, In Proceedings of ACM SIGCOMM'02, Pittsburgh, PA, USA, (Aug 2002)

4. D. A. Tran, K. A. Hua, T. Do: Zigzag: An efficient peer-to-peer scheme for media streaming, In Proceedings of IEEE INFOCOM'03, San Franciso, CA, USA, (Mar/Apr 2003)

5. Y. Chawathe: Scattercast: An Architecture for Internet Broadcast Distribution as an Infrastructure Service, Ph.D. Thesis, University of California, Berkekey, (Dec 2000)

6. P. Francis: Yoid: Extending the Internet Multicast Architecture, White Paper, http://www.icir.org/yoid

7. B. Zhang, S. Jamin, L. Zhang: Host Multicast: A Framework for Delivering Multicast to End Users, In Proceeding of IEEE INFOCOM'02, New York, NY, USA, (Jun 2002)

8. X. Zhang, J. Liu, B. Li, T. P. Yum: Data-Driven Overlay Streaming: Design, Implementation, and Experience, In Proceedings of IEEE INFOCOM'05, Miami, Florida, USA, (Mar 2005)

9. S. Banerjee, S. Lee, B. Bhattacharjee, A. Srinivasan: Resilient multicast using overlays, In Proceedings of ACM SIGMETRICS'03, San Diego, CA, USA, (Jun 2003)

10. P. Eugster, R. Guerraoui, A.M. Kermarrec, L. Massoulie: From epidemics to distributed computing, IEEE Computer, (2004) 37(5):60-67

11. S. Jin and A. Bestavros: Cache-and-relay streaming media delivery for asynchronous clients, In Proceedings of NGC'02, Boston, MA, USA, (Oct 2002)

12. Y. Cui, B. Li, K. Nahrstedt: oStream: asynchronous streaming multicast, IEEE Journal on Selected Areas in Communications, (2004) 22(1): 91-106 